\title{
Guidelines for the Doppler Assessment of the Umbilical and Middle Cerebral Arteries in Obstetrics
}

\author{
${ }^{1}$ Autumn Broady, ${ }^{2}$ Ivica Zalud
}

\begin{abstract}
The use of Doppler ultrasound is an important tool in the obstetrical assessment of an at-risk fetus. Principles of the Doppler effect can be used to monitor placental and fetal blood flow in pregnancies complicated by fetal growth restriction or in the surveillance for fetal anemia from maternal alloimmunization. Indications and techniques for umbilical artery and middle cerebral artery Doppler assessment are reviewed.
\end{abstract}

Keywords: Doppler ultrasound, Middle cerebral artery, Pregnancy, Umbilical artery.

How to cite this article: Broady A, Zalud I. Guidelines for the Doppler Assessment of the Umbilical and Middle Cerebral Arteries in Obstetrics. Donald School J Ultrasound Obstet Gynecol 2016;10(4):418-421.

Source of support: Nil

Conflict of interest: None

\section{UMBILICAL ARTERY}

Blood flow in the umbilical cord is usually high flow and low resistance with increasing end-diastolic flow as gestation advances (Figs 1A and B). ${ }^{1}$ This low-impedance system can be seen as early as 14 weeks' gestation. $^{2}$ The umbilical arteries carry deoxygenated blood from the fetus to the placenta, and changes in their blood flow are indicative of placental compromise. Doppler evaluation of the umbilical artery is to be performed in the presence of fetal intrauterine growth restriction (IUGR), as it has not been shown to be a reliable screening tool in the prediction of development of IUGR. ${ }^{3}$

A biphasic waveform corresponding to systole and diastole is produced when the umbilical artery is evaluated by pulsed Doppler. ${ }^{4}$ Forward flow of blood should occur during both phases of the cardiac cycle. Qualitative assessment of the direction of end-diastolic flow in the umbilical artery is a reproducible method to evaluate the presence of placental compromise, particularly in the

\footnotetext{
${ }^{1}$ Fellow and Clinical Instructor, ${ }^{2}$ Professor and Chair

1,2Department of Obstetrics and Gynecology, Women's Health John A Burns School of Medicine, University of Hawaii, Honolulu Hawaii, USA

Corresponding Author: Autumn Broady, Fellow and Clinical Instructor, Department of Obstetrics and Gynecology, Women's Health, John A Burns School of Medicine, University of Hawaii Honolulu, Hawaii, USA, e-mail: abroady@hawaii.edu
}

setting of IUGR and placental insufficiency. Reduction followed by reversal of end-diastolic blood flow in the umbilical artery has been shown to correlate with the degree of placental dysfunction, particularly the obliteration of small arteries in the tertiary stem villi. Reversed flow represents more advanced dysfunction, with more than $70 \%$ of placental arteries compromised. ${ }^{2,5}$

The most commonly used quantitative assessments of the umbilical artery using Doppler are the pulsatility index and systolic/diastolic (S/D) ratio. ${ }^{3}$ These ratios are independent of the alterations of measured blood flow due to changes in the angle of insonation of the ultrasound beam. ${ }^{4}$ The pulsatility index should be used in cases of absence of end-diastolic flow as the S/D will be immeasurable.

Standard technique should be employed in obtaining the umbilical artery waveform to ensure reproducibility and accuracy of measurements (Table 1). Doppler measurements should be taken from a free-floating loop of umbilical cord near the abdominal insertion site. Care should be taken to ensure the loop of cord is not compressed between the uterine wall and the fetus, as this can alter cord resistance and blood flow. Measurements taken near the placental insertion have a lower resistance than sites close to the abdominal insertion. ${ }^{6}$ Measurements should be taken in the absence of fetal breathing and while the waveform is uniform. The umbilical artery is identified on color Doppler using the number of vessels identified, pulsatile color flow pattern, caliber, and blood flow direction. ${ }^{7}$ The pulsed Doppler gate should be placed over the targeted area of the umbilical artery and the gate should be sized to ensure that only the artery is sampled.

Table 1: Methodological guidelines for Doppler assessment of the umbilical artery blood flow

- Locate a free loop of uncompressed cord.

- Identify umbilical artery, using color Doppler as necessary.

- Magnify until the loop of cord fills the majority of the image.

- Pulsed Doppler gate 1-2 mm.

- Adjust caliper gate over single umbilical artery.

- Adjust power Doppler scale to fit velocity.

- Obtain at least 5 uniform waveforms for measurement of indices.

- Ensure absence of fetal breathing and movement during measurement.

- Observe the As Low as Reasonably Achievable (ALARA) principle during evaluation. 

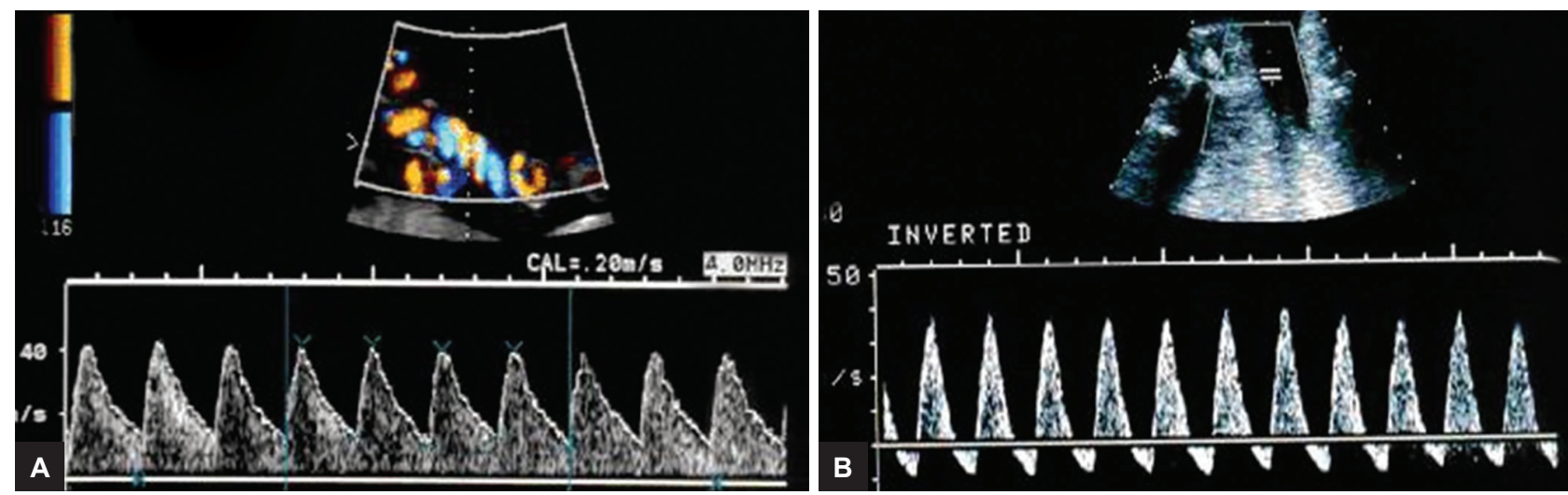

Figs 1A and B: Doppler waveform of the umbilical artery: (A) Normal umbilical artery waveform; and (B) abnormal (reversed) end-diastolic waveform
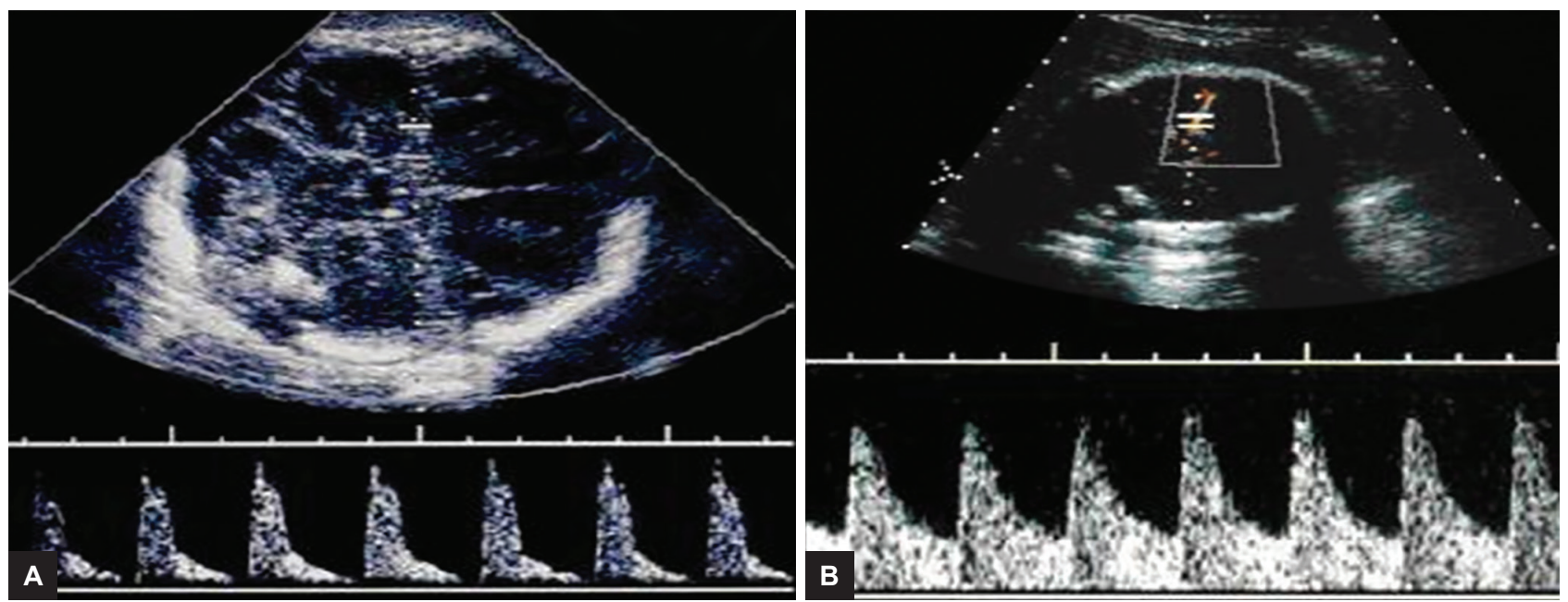

Figs 2A and B: Doppler waveform of the MCA: (A) Normal MCA Doppler waveform; and (B) abnormal MCA Doppler waveform

Adjustments in the gain and pulsed Doppler scale should be made to ensure the flow velocity is within the measured scale. The angle of insonation should be kept as low as possible to maximize systolic and diastolic flow. ${ }^{7}$

\section{MIDDLE CEREBRAL ARTERY}

In contrast to the umbilical artery, fetal cerebral circulation is a high-impedance system (Figs 2A and B). The middle cerebral artery (MCA) is the most accessible cerebral vessel to visualize via ultrasound and carries the majority of cerebral fetal blood. ${ }^{8}$ The MCA is a terminal branch of the carotid artery, and arises from the circle of Willis within the fetal brain.

A biphasic waveform corresponding to systole and diastole is produced when the MCA is evaluated by pulsed Doppler. ${ }^{4}$ Forward flow of blood should occur during both phases of the cardiac cycle. Commonly used quantitative measurements of blood flow in the MCA include the peak systolic velocity (PSV) and resistance index. Currently, the assessment of and screening for fetal anemia in pregnancies complicated by maternal alloimmunization or congenital infections (e.g., parvo virus) is the accepted use of MCA Doppler evaluation. Normally,
PSV increases with advancing gestation, necessitating the conversion of values of multiples of the median (MoM) to evaluate for pathological increases. In addition, PSV increases in the presence of fetal anemia due to increased cardiac output and lower blood viscosity. ${ }^{4}$

Measurement of cerebral blood flow has also been investigated in fetal growth restriction, based on the physiologic redistribution of blood flow that occurs in the presence of fetal hypoxemia. Hypoxemia results in fetal adaptations in blood flow, with the preservation of flow to the brain, heart, and adrenal glands, producing the so-called "brain-sparing" effect.9,10 The cerebroplacental ratio (CPR) is calculated by dividing Doppler indices of the MCA by the indices of the umbilical artery. The pulsatility index is the most commonly used index in calculating the CPR, although other indices have been studied. This ratio accounts for the interaction of blood flow changes in the fetal brain as a result of increased placental resistance. ${ }^{11}$ Abnormal CPR values $<1$ are typically defined as abnormal, although conversion of values to multiples of the median has also been reported. Recently, abnormal CPR values have been associated with increased risk of adverse perinatal outcomes, including 
Table 2: Methodological guidelines for Doppler assessment of the middle cerebral artery blood flow (adapted from SMFM ${ }^{15}$ )

- Obtain a transverse view of the fetal head at the level of the sphenoid bones. Brain landmarks include visualization of the thalami and cavum septum pellucidum.

- Visualize the circle of Willis using color Doppler.

- Visualize the middle cerebral artery originating off the circle of Willis. The entire length of the MCA should be seen.

- Select MCA closest to the transducer if feasible.

- Magnify the image such that the MCA occupies $>50 \%$ of the image.

- Pulsed Doppler gate 1-2 mm.

- Adjust caliper gate over MCA. The PSV should be sampled close to its origin from the internal carotid artery.

- Adjust pulse-wave Doppler scale to fit velocity.

- The angle between the ultrasound beam and the direction of blood flow should be as close to $0^{\circ}$ as possible, ideally $<10^{\circ}$, and parallel to the entire vessel length. If this is not possible, the angle of insonation should be $<30^{\circ}$.

- Obtain at least 5 uniform waveforms for measurement of PSV. The highest waveform should be used to measure the PSV.

- Ensure absence of fetal breathing and movement during measurement.

- Repeat sequence at least 3 times. The highest PSV value should be used for clinical care.

- Observe the As Low as Reasonably Achievable (ALARA) Principle during evaluation.

stillbirth, perinatal mortality, fetal distress, neonatal intensive care admission, and poor neonatal neurologic outcomes. ${ }^{11-14}$ Further investigation is needed before MCA assessment is incorporated into standard clinical practice in the setting of fetal growth restriction.

Precise methodology is paramount in measurement of MCA indices. The Society for Maternal-Fetal Medicine recently published step-by-step guidelines for the proper technique of measurement of the MCA PSV, which are reviewed in detail in Table 2 . The measurements should be performed when the fetus is at rest and MCA measurements should be repeated at least three times to minimize effects of fetal heart rate change on MCA indices. ${ }^{15}$ Care should also be taken not to put excessive pressure on the fetal head during the measurement as this may falsely reduce end-diastolic flow. ${ }^{7}$ Measurements should be taken from the proximal portion of the MCA, near its origin at the circle of Willis, as PSV decreases with increasing distance from the origin. ${ }^{7}$ The angle of insonation should be as close to $0^{\circ}$ as possible for most accurate estimations of blood flow velocity, although angle correction is possible and accurate if fetal positioning does not allow for this angle. ${ }^{16}$ The highest point of the pulsed Doppler waveform is then measured to obtain the PSV and other indices.

\section{REFERENCES}

1. Fleischer A, Schulman H, Farmakides G, Bracero L, Blattner P, Randolph G. Umbilical artery waveforms and intrauterine growth retardation. Am J Obstet Gynecol 1985 Feb;151(4): 502-505.

2. Trudinger BJ, Stevens D, Connelly A, Hales JR, Alexander G, Bradley L, Fawcett A, Thrompson RS. The effect of embolizations of the umbilical circulation. Am J Obstet Gynecol 1987 Dec;157(6):1443-1448.

3. Society for Maternal-Fetal Medicine Publications Committee, Berkley E, Chauhan SP, Abuhamad A. Doppler assessment of the fetus with intrauterine growth restriction. Am J Obstet Gynecol 2012 Apr;206(4):300-308.

4. Callen, P.W. Ultrasonography in obstetrics and gynecology. Philadelphia, PA: Elsevier; 2008.

5. Thompson RS, Trudinger BJ. Doppler waveform pulsatility index and resistance, pressure and flow in the umbilical placental circulation: an investigation using a mathematical model. Ultrasound Med Biol 1990;16(5):449-458.

6. Trudinger, BJ. Doppler ultrasonography and fetal well being. In: Reece, EA.; Hobbins, JC.; Mahoney, M.; Petrie, RH., editors. Medicine of the fetus and mother. Philadelphia, PA: JB Lippincott Co; 1992.

7. Creasy, RK.; Resnik, R.; Iams, JD.; Lockwood, CJ.; Moore, TR.; Greene, MF. Maternal-fetal medicine principles and practice. Philadelphia, PA: Elsevier; 2014.

8. Veille JC, Hanson R, Tatum K. Longitudinal quantitation of middle cerebral artery blood flow in normal human fetuses. Am J Obstet Gynecol 1993 Dec;169(6):1393-1398.

9. Mari G, Deter RL. Middle cerebral artery flow velocity waveforms in normal and small-for-gestational age fetuses. Am J Obstet Gynecol 1992 Apr;166(4):1262-1270.

10. Behrman RE, Lees MH, Peterson EN, De Lannoy CW, Seeds AE. Distribution of the circulation in the normal and asphyxiated fetal primate. Am J Obstet Gynecol 1970 Nov;108(6):956-969.

11. DeVore GR. The importance of the cerebroplacental ratio in the evaluation of fetal well-being in SGA and AGA fetuses. Am J Obstet Gynecol 2015 Jul;213(1):5-15.

12. Kahlil AA, Morales-Rosello J, Elsaddig M, Khan N, Papageorghiou A, Bhide A, Thilaganathan B. The association between fetal Doppler and admission to the neonatal unit at term. Am J Obstet Gynecol 2015 Jul;213(1):57.e1-7.

13. Kahlil A, Morales-Rosello J, Townsend R, Morlando M, Papageorghiou A, Bhide A, Thilaganathan B. Are fetal cerebroplacental ratio and impaired placental perfusion recorded in the third trimester predictors of stillbirth and perinatal loss? Ultrasound Obstet Gynecol 2016 Jan;47(1): 74-80.

14. Jugović D, Tumbri J, Medić M, Jukić MK, Kurjak A, Arbeille P, Salihagic-Kadić A. New Doppler index for prediction of perinatal brain damage in growth-restricted and hypoxic fetuses. Ultrasound Obstet Gynecol 2007 Sep;30(3):303-311. 
15. Society for Maternal-Fetal Medicine, Mari G, Norton ME, Stone J, Berghella V, Sciscione AC, Tate D, Schenone MH. SMFM Clinical Guideline \#8: the fetus at risk for anemia-diagnosis and management. Am J Obstet Gynecol 2015 Jun;212(6):697-710.
16. Ruma MS, Swartz AE, Kim E, Herring AH, Menard MK, Moise KJ Jr. Angle correction can be used to measure peak systolic velocity in the fetal middle cerebral artery. Am J Obstet Gynecol 2009 Apr;200(4):397.e1-3. 